\title{
Effectiveness Of Cognitive Behaviour Therapy in managing Rumination And Insomnia among adolescent girls
}

\author{
Chandramohan. $\mathrm{P}^{1}$, N. V. Amudha Devi ${ }^{2}$ \\ ${ }^{1}$ Inspector, CBCID Q Branch, Tamil Nadu. \\ ${ }^{2}$ Assistant Professor, Department of Psychology, Avinashilingam Institute for Home Science and Higher \\ Education for Women, Coimbatore. \\ Corresponding author: N.V. Amudha Devi \\ Email - amudhadevikrishnan@gmail.com
}

\begin{abstract}
Background: The present study attempts to study the effectiveness of cognitive behaviour therapy in managing rumination and insomnia among adolescent girls from the Avinashilingam Institute for Home Science and Higher Education for Women, Coimbatore.

Methodology: 200 students from undergraduates were screened for their level of Rumination and Insomnia using Ruminative Response Scale and Insomnia Inventory. Out of 200, 120 students with high and average level of Rumination and Insomnia were selected using random sampling technique. Cognitive Behaviour Therapy was given to manage Rumination and reduce Insomnia for the adolescent girls.

Results: The age range is 17 to 18 years. Pearson correlation, mean and standard deviation and ' $t$ ' test was computed to analyse the data. The results indicate that there was a positive correlation between rumination and insomnia and significant difference in managing rumination and insomnia among the adolescent girls proving the effectiveness of Cognitive Behaviour Therapy.

Conclusion: Cognitive Behaviour Therapy is an effective therapy for the management of insomnia and rumination in adolescent girls and further studies in this area are needed.
\end{abstract}

Keywords: Rumination, Insomnia, Cognitive Behaviour Therapy

(Paper received $-17^{\text {th }}$ November 2020 , Peer review completed $-20^{\text {th }}$ December 2020 )

(Accepted $-28^{\text {th }}$ December 2020)

\section{INTRODUCTION}

In the current scenario, the adolescents are vulnerable to many issues involving all aspects such as psychological, social and biological changes. Repetitive thoughts and sleep disturbances are very common these days. But when it gets much engrossed and crosses the extent on prolongation will lead to mental health issues and Insomnia. Therefore, it is essential to make note on these uncommon symptoms and to do the needful. Jersild defined Adolescence is a span of fears during this period move from childhood to adulthood mentally, emotionally, socially and physically". In India Adolescence is a phase that is more difficult to define, particularly in terms of age. The classic age wise grouping for Adolescence is between 11 to 18 years for girls and between 12 to 18 years for boys [1].

Rumination and sleep quality was explored in a study and there were some interactions between sleep quality, depression, and rumination among adolescents [2]. Sleep quality was generally related to depression and also it was found that there was relationship exist between insomnia, general rumination and depression. Conway and other have defined rumination "consists of repetitive thoughts concerning one's present distress and the circumstances surrounding the sadness" [3].

Rumination has several features such as -

- It is unintended thoughts and it's difficult to eliminate 
- Rumination has been found to be associated with a number of cognitions, affective and behavioral component

- Rumination involved in the sense of illogical

There are two forms of ruminative thinking were distinguished and identified as the two distinct rumination components, such as Brooding (referring to self-critical moody pondering) and Reflection (capturing emotionally neutral pondering distinguished [4]. Recent studies suggested that brooding represents the more maladaptive component of rumination; reflection, on the other hand, appears a more adaptive form of rumination [4-5]. According to American Psychiatric Association, found that in the primary care $10-20 \%$ of people had significant sleep problems, one-third of adults reported insomnia symptoms and $6-10 \%$ meet the criteria for insomnia disorder [6]. The general consensus based on a many population studies is that one third of adults had frequent trouble falling sleep, staying sleep or overall poor sleep quality. Especially in Adolescents and young adults experienced more sleep disturbances at this point in their lives than an an previous developmental stage. This population tends to experience sleep phase syndrome (staying up late and sleeping late), irregular sleep patterns and an increased risk of insomnia and other sleep disturbances [7].

Insomnia is defined as a general difficulty initiating or maintaining sleep that lasts for weeks at a time and is usually marked by significant distress or impairment in normal functioning [8-9]. The American Psychological Association defined that there are two types of Insomnia i.e. Primary and Secondary and Acute versus Chronic Insomnia.

Primary Insomnia: Primary insomnia means that a person is having sleep problems that are not directly associated with any other health condition or problem.

\section{Criteria for Primary Insomnia [10]}

- It is predominantly difficult in initiating and maintaining sleep.

- The sleep disturbance (or associated with daytime fatigue) causes significantly distress or impairment in social, occupational or other important areas.

- Attention and concentration problems, negative mood, social, occupational and vocational dysfunction.

Secondary Insomnia: It means that a person is having sleep problems because of something else, such as a health condition (like asthma, depression, arthritis, cancer, or heartburn); pain; medication they are taking; or a substance they are using (like alcohol).

Acute versus Chronic Insomnia: Insomnia also varies in how long it lasts and how often it occurs. It can be short term (acute insomnia) or can last a long time (chronic insomnia). It can also come and go, with periods of time when a person has no sleep problems. Acute insomnia can last from one night to a few weeks. Insomnia is called chronic when a person has insomnia at least three nights a week for a month or longer.

Sleep difficulties are linked to both physical and emotional problems. Sleep problems can both contribute to or exacerbate mental health conditions and be a symptom of other mental health conditions. The poor quality of sleep has many potential consequences. The most obvious concerns are fatigue, decreased energy, irritability, problems focusing, the ability to make decisions and mood can also be affected, sleep problems often coexist with symptoms of depression or anxiety. Lack of sleep and too much sleep are linked to many chronic health problems such as heart disease and diabetes.

Rumination is a common issue for people with depression as well as most important issue with the people of insomnia [11-13]. Rumination is a form of repetitive thinking. The content of thought differs slightly in those with insomnia [13]. The repeated past focused thoughts are causes of the fatigue in previous night sleep. Specifically, two types of repetitive thinking have been proposed to play an important role insomnia: worry and rumination [14]. Worry is a coping strategy that consists of engaging in abstract repetitive verbal thinking focused on uncertain events with a potential future negative outcome [15-16]. Rumination, on the other hand, is a coping strategy that consists of abstract repetitive thinking on the causes and consequences of negative emotions [17].

Cognitive Behaviour Therapy has been shown to be an effective way of treating a number of different mental health conditions. In addition to depression or anxiety disorders, Cognitive Behaviour Therapy can also 
help people with - Obsessive Compulsive Disorder, Panic Disorder, Post Traumatic Stress Disorder, Phobias, Eating disorders - such as Anorexia and Bulimia, Sleep problems - such as Insomnia.

Lin and others [19] conducted a study on the mediating effects of repetitive negative thinking in the relationship between perfectionism and adolescent sleep quality. One thousand six hundred and sixty-four Chinese adolescents with a mean age of 15.0 years were selected and administered by four measures relating to perfectionism, sleep quality, worry, and rumination. The results showed that maladaptive perfectionism was positively correlated with poor sleep quality in adolescents, which was mediated by both worry and rumination. However, adaptive perfectionism was not significantly associated with adolescent sleep quality, and this relationship was suppressed by rumination.

Harding and Mezulis [19] investigated on whether the rumination is a risk and a protective factor. The sample consisted of 321 adults first compared three confirmatory factor analysis models of the relationship between brooding and positive rumination. They utilized structural equation modelling to examine whether brooding and positive rumination mediated the relationship between trait positive affect and depressive symptoms, controlling for baseline depressive symptoms, trait negative affect), and the distinct effects of each mediator. Results supported a conceptualization of brooding and positive rumination as distinct but related constructs, represented as a common process of affect amplification to explain how rumination may amplify resilience or risk in predicting depressive symptoms. Furthermore, positive rumination and brooding were distinctly predicted by trait positive effect, suggesting that trait positive affect exerts distinct effects on protective and risk forms of rumination. Less brooding mediated the relationship between greater trait positive affect and fewer depressive symptoms but positive rumination did not.

Conroy and others [20] assessed five week's insomnia treatment in adolescents with major depressive disorder and insomnia. This was an open label trial of a modified Group Cognitive Behaviour Therapy for Insomnia. Adolescents with major depressive disorder $(n=16$; mean age $=17.3+/-1.7)$ were administered by the Children's Depression Rating Scale and an Insomnia Severity Index, Quick Inventory of Depressive Symptomatology and Multidimensional Fatigue Inventory. The results showed that decreased wake during the night as per the Daily Sleep Diaries, increased sleep time, and increased quality of sleep improved across treatment.

Tavernier and his team [21] examined in relation to four objectively measured sleep outcomes (sleep hours; sleep latency; sleep efficiency; and length of wake bouts) among adolescents. The participants ( $\mathrm{N}=77 \mathrm{high}$ school students; $42.9 \%$ female; $\mathrm{M}=14.37$ years) wore an act watch and completed daily diaries for 3 days. The results of hierarchical linear models (controlling for age, gender, race, ethnicity, parental employment status, income, puberty and caffeine) indicated that negative social evaluative emotions and high arousal affective experiences generally predicted poor sleep outcomes, whereas low arousal affective experiences were associated with good sleep outcomes. The results highlighted the detrimental effects of negative social evaluative emotions and high arousal affective states for adolescent sleep.

Nota and Coles [22] examined the relation between Repetitive negative thinking and the timing of sleep and both disorder specific measures of a Repetitive negative thinking. One hundred undergraduates were selected for the study. Replicating prior findings, shorter sleep duration was cross sectional associated with more rumination and delayed sleep timing was associated with more obsessive-compulsive symptoms. Further, extending this prior work, the transdiagnostic measure of Repetitive negative thinking was associated with shorter sleep duration and delayed sleep timing. Individuals who endorsed a preference for later sleep and activity times also reported more Repetitive negative thinking. These findings suggested that may Repetitive negative thinking be uniquely related to both sleep duration and timing.

Yeh and others [23] examined the relationship between rumination, worry, pre sleep arousal and sleep quality. The participants were 202 young adults who completed questionnaires on sleep quality, trait rumination, and worry. After factor analysis of rumination and worry scales, three factors were found, and which were positively correlated with pre sleep cognitive arousal and sleep quality. The results of structural equation model analysis suggested that pre sleep arousal was a mediator of the effects of repetitive thought on sleep quality. Dwelling on the negative and worry engagement can predicted higher pre sleep arousal which can predict poor sleep quality. Pre sleep arousal was the key factor in the relationship between repetitive thought and sleep quality. 
The current study was designed with the following objectives -

- To assess the level of Rumination, Insomnia, and identify the relationship between Rumination and Insomnia among the participants.

- To find out the effectiveness of the Cognitive Behaviour Therapy in managing rumination and reducing insomnia among the participants.

\section{METHODOLOGY}

\section{Null hypotheses}

- There will be no correlation between Rumination and Insomnia among the participants.

- There will be no significant difference among the participants in Rumination after the administration of Cognitive Behaviour Therapy

- There will be no significant difference among the participants in Insomnia after the administration

Sample of Cognitive Behaviour Therapy

Two hundred students from Avinashilingam Institute for Home Science and higher Education for Women, Coimbatore, were initially screened for high and average level of Rumination and Insomnia. Out of 200, 120 college students with High and average Rumination and Insomnia were selected for the study and they were in the age range of 17-19 years.

Inclusion criteria

- Age range between 17-19 years

- Female students.

- Willingness to participate.

Exclusion criteria

- Below 17 years and above 19 years were excluded for the study.

- Students from other Universities were excluded.

- With any other physical disability

- Males were excluded.

Tools Used

- Case Study Schedule evolved by the investigator was used to collect the general information from the students.

- Insomnia Inventory: It was used to identify the presence of Insomnia. The inventory consists of 10 items. There are two possible responses to each item namely true or false. The respondents are asked to put a tick mark near any one of those options which suits them. There is no time limit. But the subjects are asked to respond quickly. If the sample chose more than 3 true for the given statements, then the subjects show the symptoms of Insomnia [24].

- Ruminative Responses Scale: The scale was used to measure the level of Rumination. It consists of 22 items that address how often participants engage in responses to feeling sad or depressed. It is a four-point Likert Scale with anchors of " $1=$ Never to $4=$ Always" [25].

At the onset of the research 200 students were screened from Avinashilingam Institute for Home Science and higher Education for Women, Coimbatore, by random sampling method. They were screened using Case Study Schedule, Rumination Scale and Insomnia. Among 200 students, 120 students scored high in Rumination and Insomnia was taken for the study. They were in the age range of 17-19 years. Cognitive Behavior Therapy was administered for the participants. After 15 days the Reassessment was given by using the same Questionnaires.

Before and after without control group research design was used to conduct the research. The variables were Rumination and Insomnia was measured before and after Cognitive Behaviour Therapy. 


\section{Flow Chart}

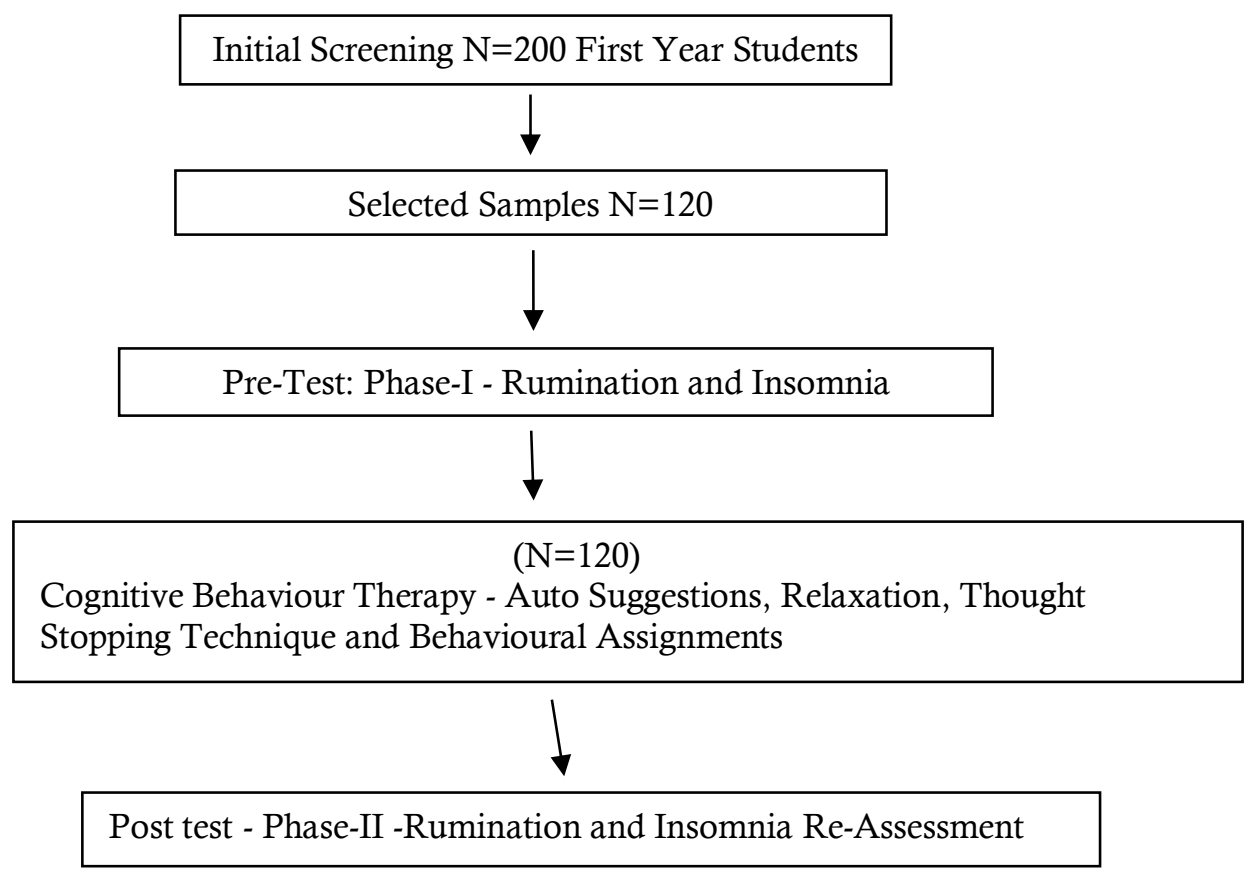

The Cognitive Behaviour Therapy includes

- Relaxation: The relaxation process goes as follows. The deep breathing exercise for 10 minutes were initially given to the students. After that the students were asked to close their eyes and were made to concentrate each organ such as hands, legs, head, neck etc. Thereby they were relaxing the whole body. This technique was given for 20 minutes.

- Thought Stopping: The thought stopping technique proves to be of great use for rumination. It helps the samples to stop the disturbing rumination thoughts. In this technique, the participants were asked to identify the recurring negative thoughts which disturb them. Then they were asked to close the eyes, breathe slowly and say STOP loudly and breathe out making them feel that the thought is going out of their body. This is repeated for five times per session.

- Auto Suggestions: Auto suggestion technique helps an individual to develop optimism in their life. In this the participants were asked to give auto suggestions to themselves such as "I am happy", "I am Healthy" etc. which also helps to overcome negativity and brings positive attitude.

- Behavioural Assignments: Behavioural assignments strengthen the effectiveness of the therapy. It is nothing but practicing the therapy at home individually on regular basis. This brings change drastically unlike practicing therapy only with therapist.

The Cognitive Behaviour Therapy was given to students in group for 6 sessions for two days in a week for one month. The therapy was given to the participants on alternate days. The duration of each session was 45 minutes to one hour.

\section{Post Assessment}

After two weeks of Cognitive Behaviour Therapy the entire participants were reassessed using Rumination and Insomnia Questionnaire.

\section{STATISTICAL ANALYSIS}

The data was analysed using SPSS package. Mean, Standard Deviation, t-test and correlation was used to ascertain the data. 


\section{RESULTS AND DISCUSSION}

Table 1: Demographic Data of the Participants $(n=120)$

\begin{tabular}{|c|c|c|c|}
\hline & Demographic Data & Number & Percent \\
\hline \multirow{2}{*}{ Age } & 17 & 61 & 51 \\
\hline & 18 & 59 & 49 \\
\hline \multirow[t]{2}{*}{ Family Type } & Joint & 38 & 32 \\
\hline & Nuclear & 82 & 68 \\
\hline \multirow[t]{2}{*}{ Place of Living } & Rural & 38 & 32 \\
\hline & Urban & 82 & 68 \\
\hline
\end{tabular}

Note: Percentages are rounded off

Table 2: Level of Rumination Before and After Cognitive Behaviour Therapy $(\mathrm{N}=120)$

\begin{tabular}{|c|c|c|c|c|c|}
\hline \multicolumn{2}{|c|}{} & \multicolumn{2}{c|}{$\begin{array}{c}\text { Before Cognitive Behaviour } \\
\text { Therapy }\end{array}$} & \multicolumn{2}{c|}{$\begin{array}{c}\text { After Cognitive Behaviour } \\
\text { Therapy }\end{array}$} \\
\hline \multirow{4}{*}{ RUMINATION } & Low & FREQUENCY & PERCENT & FREQUENCY & PERCENT \\
\cline { 2 - 6 } & Average & 55 & 0 & 62 & 52 \\
\cline { 2 - 6 } & High & 65 & 46 & 58 & 48 \\
\cline { 2 - 6 } & Total & 120 & 100 & 0 & 0 \\
\hline
\end{tabular}

Note: Percentages are rounded off

Table 3: Level of Insomnia Before and After Cognitive Behaviour Therapy ( $N=120)$

\begin{tabular}{|c|c|c|c|c|c|}
\hline \multicolumn{2}{|c|}{} & \multicolumn{2}{c|}{$\begin{array}{c}\text { Before Cognitive Behaviour } \\
\text { Therapy }\end{array}$} & \multicolumn{2}{c|}{$\begin{array}{c}\text { After Cognitive Behaviour } \\
\text { Therapy }\end{array}$} \\
\hline \multirow{3}{*}{ INSOMNIA } & Yes & FREQUENCY & PERCENT & FREQUENCY & PERCENT \\
\cline { 2 - 6 } & No & 44 & 62 & 27 & 23 \\
\cline { 2 - 6 } & Total & 120 & 38 & 93 & 77 \\
\hline \multicolumn{7}{|c|}{ Note: Percentages are rounded off } \\
\hline
\end{tabular}

Table 4: Correlation between Rumination and Insomnia $(\mathrm{N}=120)$

\begin{tabular}{|l|l|c|c|}
\hline \multicolumn{2}{|c|}{} & RUMINATION & INSOMNIA \\
\hline \multirow{4}{*}{ RUMINATION } & Pearson Correlation & 1 & $0.79^{* *}$ \\
\cline { 2 - 4 } & Sig. (2-tailed) & & 0.00 \\
\cline { 2 - 4 } & $\mathrm{N}$ & 120 & 120 \\
\hline \multirow{2}{*}{ INSOMNIA } & Pearson Correlation & $0.79^{* *}$ & 1 \\
\cline { 2 - 4 } & Sig. (2-tailed) & 0.00 & 120 \\
\cline { 2 - 4 } & $\mathrm{N}$ & 120 & \\
\hline & ${ }^{* *}$ Correlation is significant at the 0.01 level (2-tailed). \\
\hline
\end{tabular}

Table 4 indicates that the correlation value between Rumination and Insomnia of the participants is 0.78 and it is statistically significant at 0.01 level. It is highly positively correlated. Based on the above results on Rumination and Insomnia, the Hypothesis 1, "There will be no correlation between Rumination and Insomnia among the participants" is rejected.

Table 5 shows the $t$ value for before and after Cognitive Behaviour Therapy in Rumination among the participants. The results revealed that the mean Before Cognitive Behaviour Therapy was 2.54 and after Cognitive Behaviour Therapist reduced to 1.06 and the t value was 27.87 which is significant at 0.01 level. 
Hence, the Null Hypothesis 2, "There will be no significant difference among the participants in Rumination after the administration of Cognitive Behaviour Therapy" is rejected.

Table 5: Mean, Standard Deviation and $t$ value for Before and After Cognitive Behaviour Therapy in Rumination among Adolescent Girls (N=120)

\begin{tabular}{|c|c|c|c|}
\hline RUMINATION & Mean & Standard Deviation & t value \\
\hline Before & 2.54 & 0.50 & \multirow{2}{*}{$27.87^{* *}$} \\
\hline After & 1.06 & 0.42 & \\
\hline \multicolumn{4}{|c|}{${ }^{* * \text { Significant at } 0.01 \text { level }}$}
\end{tabular}

Table 6: Mean, Standard Deviation and $t$ value for Before and After Cognitive Behaviour Therapy in Insomnia among Adolescent Girls ( $\mathbf{N}=120)$

\begin{tabular}{|c|c|c|c|}
\hline INSOMNIA & Mean & Standard Deviation & t value \\
\cline { 1 - 3 } Before & 1.38 & 0.49 & \multirow{2}{*}{$5.82^{* *}$} \\
\hline After & 0.39 & 0.40 & \\
\hline
\end{tabular}

**Significant at 0.01 level

Table 6 shows the $t$ value for before and after in Insomnia among the participants. The mean score in Insomnia Before Cognitive Behaviour Therapy was 1.38 and After Cognitive Behaviour Therapist reduced to 0.39 and the ' $t$ ' value was 5.82 which is significant at 0.01 level. The participants had shown reduced level of Insomnia attributing the effect of the Cognitive Behaviour Therapy. Therefore, the Hypothesis 3, "There will be no significant difference among the participants in Rumination after the administration of Cognitive Behaviour Therapy" is rejected.

\section{CONCLUSION}

Based on the results from the above study, we can infer that the present study, Cognitive Behaviour Therapy proved to be effective in reducing rumination and insomnia among the participants.

\section{REFERENCES}

1. Jersild AT. The psychology of adolescence. The Macmillan; 1957.

2. Carney CE, Edinger JD, Meyer B, Lindman L, Istre T. Daily activities and sleep quality in college students. Chronobiol Int 2006;23(3):623-37.

3. Conway M, Csank PA, Holm SL, Blake CK. On assessing individual differences in rumination on sadness. J Personal Assess 2000;75(3):404-25.

4. Treynor W, Gonzalez R, Nolen-Hoeksema S. Rumination reconsidered: A psychometric analysis. Cogn Ther Res 2003;27(3):247-59.

5. Burwell RA, Shirk SR. Subtypes of rumination in adolescence: Associations between brooding, reflection, depressive symptoms, and coping. J Clin Child Adolesc Psychol 2007;36(1):56-65.

6. American Psychiatric Association. Sleep-Wake Disorders: DSM-5® Selections. American Psychiatric Pub; 2015.

7. Goldstein TR, Bridge JA, Brent DA. Sleep disturbance preceding completed suicide in adolescents. J Consult Clin Psychol 2008;76(1):84-9.

8. Jansson M, Linton SJ. Psychological mechanisms in the maintenance of insomnia: arousal, distress, and sleeprelated beliefs. Behav Res Ther 2007;45(3):511-21.

9. Mayers AG, Baldwin DS. The relationship between sleep disturbance and depression. Int J Psychiatr Clin Pract 2006;10(1):2-16.

10. Edinger JD, Bonnet MH, Bootzin RR, Doghramji K, Dorsey CM, Espie CA, Jamieson AO, McCall WV, Morin CM, Stepanski EJ. Derivation of research diagnostic criteria for insomnia: report of an American Academy of Sleep Medicine Work Group. Sleep 2004;27(8):1567-96.

11. Carney CE, Harris AL, Moss TG, Edinger JD. Distinguishing rumination from worry in clinical Insomnia. Behav Res Ther 2010;48(6):540-6. 
12. Carney CE, Harris AL, Moss TG, Edinger JD. Distinguishing rumination from worry in clinical characteristics and processes. Behav Res Ther 2010;21:9-16.

13. Carney CE, Edinger JD, Meyer B, Lindman L, Istre T. Symptom- focused rumination and sleep disturbance. Behav Sleep Med 2006;4:228-41.

14. Carney CE, Harris AL, Falco A, Edinger JD. The Relation between Insomnia symptoms, Mood, and Rumination about insomnia symptoms. J Clin Sleep Med 2013;9(6):567-75.

15. Borkovec TD, Robinson E, Pruzinsky T, Depree JA. Preliminary exploration of worry: Some characteristics and processes. Behav Res Ther 1983;21:9-16.

16. Borkovec TD, Ray WJ, Stober J. Worry: A cognitive phenomenon intimately linked to affective, physiological, and interpersonal behavioural processes. Cogn Ther Res 1998;22(6):561-76.

17. Nolen-Hoeksema S, Wisco BE, Lyubomirsky S. Rethinking rumination. Perspect Psychol Sci 2008;3(5):40024.

18. Lin RM, Xie SS, Yan YW, Chen YH, Yan WJ. Perfectionism and adolescent sleep quality: The mediating role of repetitive negative thinking. J Health Psychol 2017;135:910-4.

19. Harding KA, Mezulis A. Is Rumination a Risk and a Protective Factor? Eur J Psychol 2017;13(1):28-46.

20. Conroy DA, Czopp AM, Dore-Stites DM, Arnedt JT. Modified Cognitive Behavioural Therapy for Insomnia in Depressed Adolescents: A Pilot Study. Behav Sleep Med 2017;2:1-16.

21. Tavernier R, Choo SB, Grant K, Adam EK. Daily affective experiences predict objective sleep outcomes among adolescents. J Sleep Res 2016;25(1):62-9.

22. Nota JA, Coles ME. Duration and timing of sleep are associated with repetitive negative thinking. Cogn Ther Res 2015;39(2):253-61.

23. Yeh ZT, Wung SK, Lin CM. Pre-sleep Arousal as a mediator of relationships among Worry, Rumination, and Sleep Quality. Int J Cogn Ther 2013;8(1):21-34.

24. Morin CM. Measuring outcomes in randomized clinical trials of insomnia treatments. Sleep Med Rev 2003;7(3):263-79.

25. Roelofs J, Muris P, Huibers M, Peeters F, Arntz A. On the measurement of rumination: A psychometric evaluation of the ruminative response scale and the rumination on sadness scale in undergraduates. $\mathrm{J}$ Behav Ther Experiment Psychiatry 2006;37(4):299-313.

$* * * * * * * * * * * * * * * * * * * * * * * * * * * * * * * * * * * *$

Acknowledgements - We like to thank all participants who participated wholeheartedly in the study.

Conflict of Interest - Nil

Funding - Nil 